\title{
Monitoring B cell subsets and alloreactivity in kidney transplantation
}

\author{
Marta Crespo ${ }^{\mathrm{a}, \mathrm{b}, \square, 1}$ \\ mcrespo@parcdesalutmar.cat \\ Sebastiaan Heidt ${ }^{\mathrm{c}, 1}$ \\ Dolores Redondo \\ Julio Pascual ${ }^{\mathrm{a}, \mathrm{b}}$ \\ aDepartment of Nephrology, Hospital del Mar, Barcelona, Spain \\ 'Institut Hospital del Mar d’Investigacions Mèdiques), Barcelona, Spain \\ 'Dept. of Immunohaematology and Blood Transfusion, Leiden University Medical Center, Leiden, The \\ Netherlands \\ Corresponding author.
}

${ }^{1}$ Contributed equally to the manuscript authorship.

\begin{abstract}
B cells are the precursors of antibody producing plasma cells that can give rise to the formation of donorspecific antibodies. However, recent data suggest that besides their role in antibody production, B cells participate in antibody-independent responses, potentially leading to allograft rejection or allograft tolerance. The presence of $\mathrm{CD} 20^{+} \mathrm{B}$ cells in kidney graft biopsies has been shown during severe acute rejection episodes and during chronic rejection. Furthermore, operationally tolerant kidney transplant recipients showed a clear B cell dominated fingerprint of tolerance.Several techniques exist to study B cells on different levels. Numerous classification schemes allow for the distinction of many different B cell subsets using flow cytometry. Regardless, data on B cell subsets during stable graft function, rejection or tolerance remain scarce. To obtain a complete picture of the role of $\mathrm{B}$ cells during transplantation, antigen specific B cell assays may be required. Therefore, techniques have now been developed that allow for studying the specificity and frequency of HLA specific B cells.Here, we present an overview of the existent assays, panels and techniques intended to characterize peripheral B cells, and the currently available HLA specific B cell functional assays that may allow for monitoring the humoral alloimmune response in transplant recipients.
\end{abstract}

\section{Introduction}

The role of alloantibodies, especially HLA antibodies, in solid-organ transplant recipients both before and after transplantation has progressively regained attention for the last two decades. Traditionally, HLA antibodies have been considered a strong barrier for sensitized recipients to access transplantation due to their ability to induce hyperacute rejection [1]. Sensitization can occur due to pregnancy, blood transfusions, or previous transplants. The number of transfusions has decreased with the use of erythropoiesis stimulating agents, thus potentially decreasing the number of sensitized patients. However, the rate of retransplantation is increasing, as long-term graft survival improvement does not yet parallel improvement in short-term survival [2-4]. Besides, newer and more sensitive solid-phase assays are able to identify HLA antibodies otherwise undetected by cell-based techniques. Although still under intense debate [5], these tests reveal that there is a higher percentage of sensitized transplant recipients than previously identified. These transplant recipients seem to be at higher risk for antibody-mediated 
damage, which impacts early survival but also diminishes medium to long-term graft survival [6]. Huge efforts are being made to try to identify what amount, type or quality of HLA antibodies should preclude transplantation [6-9].

Additionally, post-transplant HLA donor-specific anti-HLA antibodies (DSA) are increasingly recognized as risk factors for kidney, heart, lung, pancreas and liver allograft loss [10-18]. Intragraft donor-specific antibodies have been obtained from biopsies in transplant recipients with circulating DSA and microvascular inflammation, supporting their pathogenic role in graft damage[19]. Detection of DSA in serum, together with typical histological changes of microvascular damage leads to the diagnosis of antibody-mediated rejection[20-22]. This has arisen as a major pathological entity responsible for early graft loss, mostly in sensitized patients, and for over $50 \%$ long-term censured graft loss as a consequence of chronic inflammation and progressive tissue destruction[23-25].

Important questions, such as whether the detection of different levels or distinct avidity of HLA DSA can predict specific graft outcome are not answered yet. Furthermore, it is unclear whether the absence of DSA reflects absence of a B cell response to the donor, active tolerance or a masked immune response by binding of DSA to the graft [26].

Recent data suggest that besides their role in antibody production, B cells participate in antibodyindependent responses, potentially leading to allograft rejection or allograft tolerance [23,27,28]. The transplant community has therefore widened their focus to B cells as important regulators of alloimmune responses by their ability to induce donor-specific damage through DSA[11-14,23,29].

Several tools are currently available to study HLA alloantibodies and general B-cell properties, but assays to study alloantigen specific B-cells are still a matter of research. Studies on B cells in transplantation have been hampered by the heterogeneity of subsets that may participate in responses to the graft and antibody production and extremely low frequencies of peripheral antigen-specific B cells. Here, we present an overview of the existent assays and techniques intended to describe characteristics of peripheral B cells and the currently available HLA specific B cell functional assays that may allow for monitoring the humoral alloimmune response in transplant recipients. Although the main focus of this review is on the kidney transplant setting, the techniques described herein will be applicable to other transplant settings as well.

\section{B cell immunophenotyping}

\subsection{B cell subsets}

In the $70 \mathrm{~s}, \mathrm{~B}$ and $\mathrm{T}$ cells were first identified as two different lymphocyte populations [30]. B cells are known as lymphocytes that play a key role in the humoral immune response. In this response, B cells provide protection by producing immunoglobulin molecules, also known as antibodies, which specifically target antigens associated with invading microbes. B cells follow a complex maturation program to express immunoglobulin molecules capable of recognizing and binding the virtually unlimited number of antigens present in the external environment. B cell development proceeds through several intermediate stages that can be distinguished on basis of the expression of various cell surface markers.

Immature B cells leave the bone marrow as transitional B cells that are typically short-lived and functionally immature and become fully mature naïve B cells after colonizing peripheral lymphoid organs. Naïve B cells undergo differentiation after encountering antigen in secondary lymphoid organs such as spleen, lymph nodes, and mucosa-associated lymphoid tissue. Upon recognition of protein antigens by their surface receptor (B cell receptor; BCR), naïve B cells either move to extra-follicular 
foci, where they differentiate into short-lived IgM secreting plasmablasts, or move to germinal centers, areas that stimulate clonal expansion, immunoglobulin gene diversification and isotype switching. In the dark zone of the germinal center, B cells undergo proliferation and become centroblasts. In the light zone of the germinal center, centroblasts differentiate into centrocytes that undergo selection and differentiation into plasma cells, which migrate to the bone marrow, or memory B cells, which recirculate to survey peripheral lymphoid organs for the presence of antigen [31].

During the last two decades, multicolor flow cytometry has allowed the identification of multiple subsets of functionally distinct B cells in humans, including transitional B cells, naïve B cells, plasma cells and memory B cells. The analysis of human B cell populations by flow cytometry has mainly been based on the expression of four cell surface markers: CD19, IgD, CD38 and D27. Two major categorization schemes have been put forward depending on the relative expression of either $\operatorname{IgD}$ and $\mathrm{CD} 38$ or $\operatorname{IgD}$ and CD27 [32].

A first modern classification of five B cell subpopulations came from studies on tonsillar B cells considering surface expression of $\operatorname{IgD}$ and CD38 [33]. This so called Bm1-Bm5 classification includes [34] (Fig. 1A):

Bm1: virgin naïve cells $\left(\mathrm{IgD}^{+} \mathrm{CD} 38^{-}\right)$

Bm2: activated naïve cells $\left(\operatorname{IgD}^{+} \mathrm{CD} 38^{+}\right)$

$\mathrm{Bm}^{\prime}$ : pre-germinal center $(\mathrm{GC})$ cells $\left(\mathrm{IgD}^{+} \mathrm{CD} 38^{++}\right)$

$\mathrm{Bm} 3+4$ : germinal center $(\mathrm{GC})$ cells $\left(\mathrm{IgD}^{-} \mathrm{CD} 38^{++}\right)$

Bm3: in centroblasts

Bm4: in centrocytes

Bm5: memory cells $\left(\mathrm{IgD}^{-} \mathrm{CD} 38^{++}\right)$: early or late depending on the expression of $\mathrm{CD} 38$

Early Bm5: CD38+

Late Bm5: CD38- 

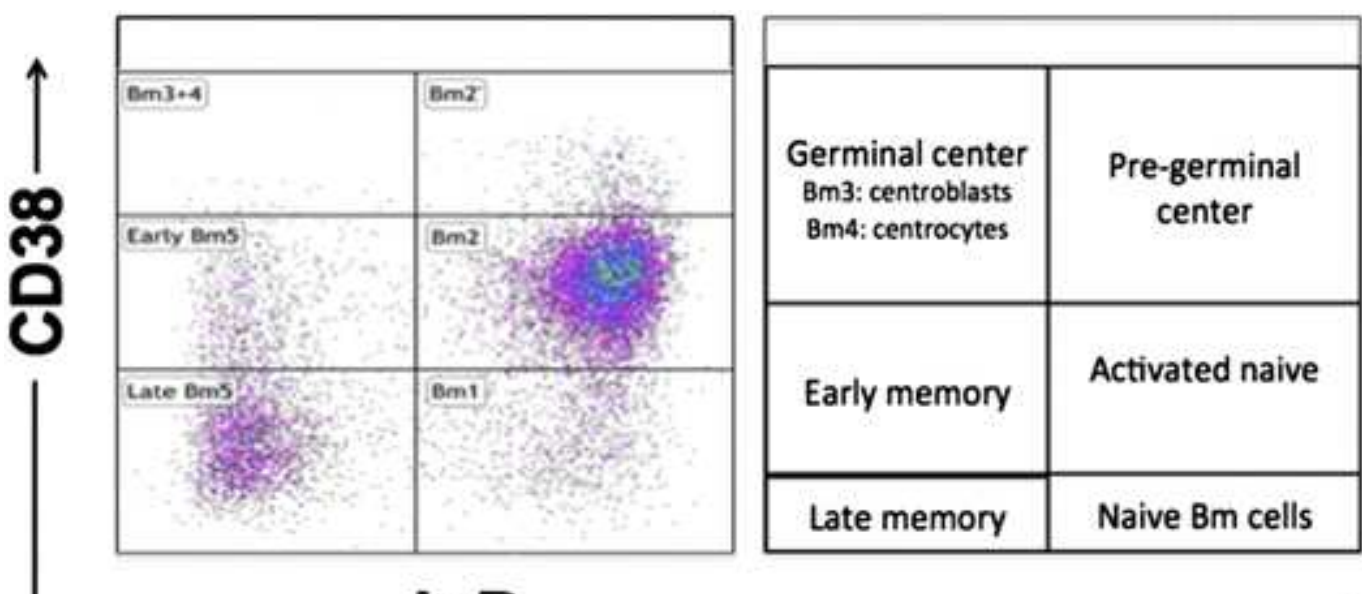

A

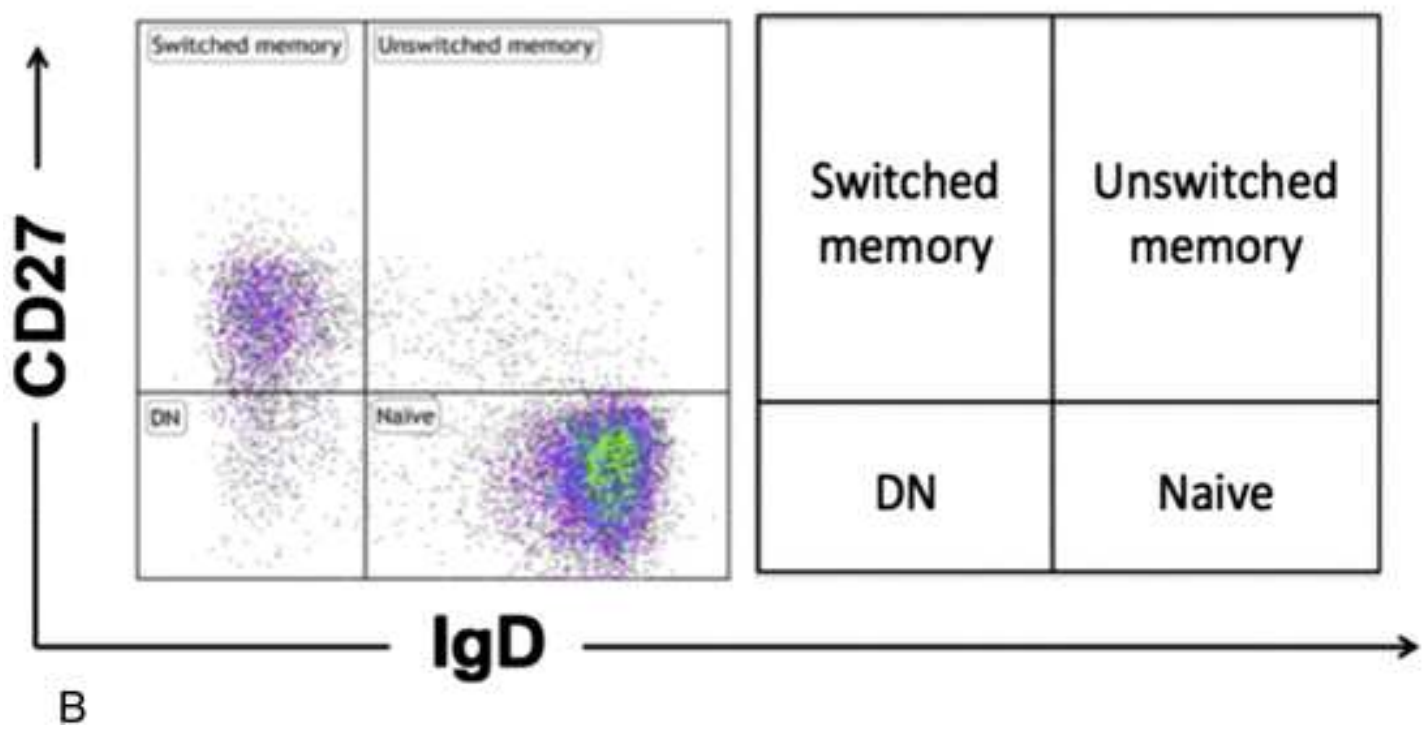

Fig. 1 Classification of human B cell subsets based on the expression of IgD/CD38.

Bohnhorst et al. confirmed that peripheral B cell subpopulations could follow this Bm1-Bm5 classification with the exception of $\mathrm{Bm} 3$ and $\mathrm{Bm} 4$ composed of germinal center cells [35]. This scheme has some limitations, as peripheral $\mathrm{Bm} 1$ and $\mathrm{Bm} 2$ could include unswitched memory cells and $\mathrm{Bm} 2^{\prime}$ in the periphery are mainly transitional B cells.

An alternative classification method, based on IgD/CD27 staining, relies on the fact that CD27 is a marker for memory B cells so that it discriminates memory B cells $\left(\mathrm{CD} 27^{+}\right)$and naïve $\mathrm{B}$ cells $\left(\mathrm{CD} 27^{-} \mathrm{IgD}^{+}\right)[36,37]$. Considering these markers, B cells can be grouped in (Kaminski. Front I. 2012) (Fig. 1B):

\begin{tabular}{|l|l|}
\hline Double-negative memory & $\operatorname{IgD}^{-} \mathrm{CD} 27^{-}$ \\
\hline Non-switched memory & $\operatorname{IgD}^{+} \mathrm{CD} 27^{+}$ \\
\hline IgM-only memory & $\operatorname{IgM}^{+} \operatorname{IgD}^{-} \mathrm{CD} 27^{+}$ \\
\hline Switched memory & $\operatorname{IgM}^{-} \operatorname{IgD}^{-} \mathrm{CD} 27^{+}$ \\
\hline
\end{tabular}


In this model, memory $\mathrm{CD} 27^{+} \mathrm{B}$ cells are split into those $\mathrm{IgD}^{+}$, usually $\operatorname{IgM}^{+}$, and those $\operatorname{IgD}^{-}$or switched-memory B cells, mostly expressing IgG or IgA, and a small proportion of memory cells expressing only IgM. Also here, some controversy exists, since CD27- memory B cells have also been described [38].

Human B cell subsets can alternatively be defined by their surface expression levels of CD24 and CD38 (naïve, memory and transitional B cells)[39]. Naïve B cells are CD24 ${ }^{\mathrm{dim}}$ CD $38^{\mathrm{dim}}$, whereas memory $\mathrm{B}$ cells are $\mathrm{CD} 24^{\mathrm{hi}} \mathrm{CD} 38^{-}$. Transitional B cells are characterized by high expression levels of CD24 and CD38, which can be supplemented by the addition of CD27 negativity and CD24 positivity.

The way how subsets defined by one classification scheme relate to those subsets defined by other scheme is not often clear. In an effort to combine the above classifications, Sanz et al. worked on six different 10-color flow cytometry panels according to the phenotypic complexity of B cells with antibodies against CD3, CD19, IgD, IgM, IgG, CD10, CD24, CD27, CD38, CD1c, CD45 (B220) and FcRH4. The analysis of additional surface markers permits the identification of additional subpopulations of memory cells within both $\mathrm{CD} 27^{+}$and $\mathrm{CD} 27^{-}$cells[32]. Table 1 shows a simplified scheme adapted from this classification emphasizing the combination of $\operatorname{IgD}, \mathrm{CD} 27$ and CD38 (Table 1).

Table 1 The phenotypes of human memory B cell subsets in the peripheral blood (adapted fromSanz et al.).

\begin{tabular}{|c|c|c|c|}
\hline & $\operatorname{IgD}$ & CD27 & CD38 \\
\hline Unswitched memory & $\operatorname{IgD}+$ & $\mathrm{CD} 27+$ & $\begin{array}{l}\text { CD38 - } \\
\text { CD38 + }\end{array}$ \\
\hline Switched memory & $\operatorname{IgD}-$ & $\mathrm{CD} 27+$ & $\begin{array}{l}\text { CD38 - } \\
\text { CD38 + }\end{array}$ \\
\hline CD27-memory & $\operatorname{IgD}-$ & $\mathrm{CD} 27-$ & $\begin{array}{l}\text { CD38 - } \\
\text { CD38 + }\end{array}$ \\
\hline Early Bm5 memory & $\operatorname{IgD}-$ & $\begin{array}{l}\mathrm{CD} 27+ \\
\mathrm{CD} 27-\end{array}$ & $\mathrm{CD} 38+$ \\
\hline Late Bm5 memory & $\operatorname{IgD}-$ & $\begin{array}{l}\mathrm{CD} 27+ \\
\mathrm{CD} 27-\end{array}$ & CD38 - \\
\hline
\end{tabular}

A "Standardizing immunophenotyping for the Human Immunology Project" has recently been started to homogenize the immunophenotype so that monitoring by flow cytometry could be useful in several clinical settings. This project acknowledges the impossibility to work with all possible B cell markers and proposes to work with an eight color antibody panel with CD19 or CD20 for B cells, CD38 for plasmablasts and transitional B cells, CD24 for transitional B cells and IgD/CD27 for naïve and memory B cells (Fig. 2) [40]. 
$\mathrm{CD}_{19}{ }^{+}$cells

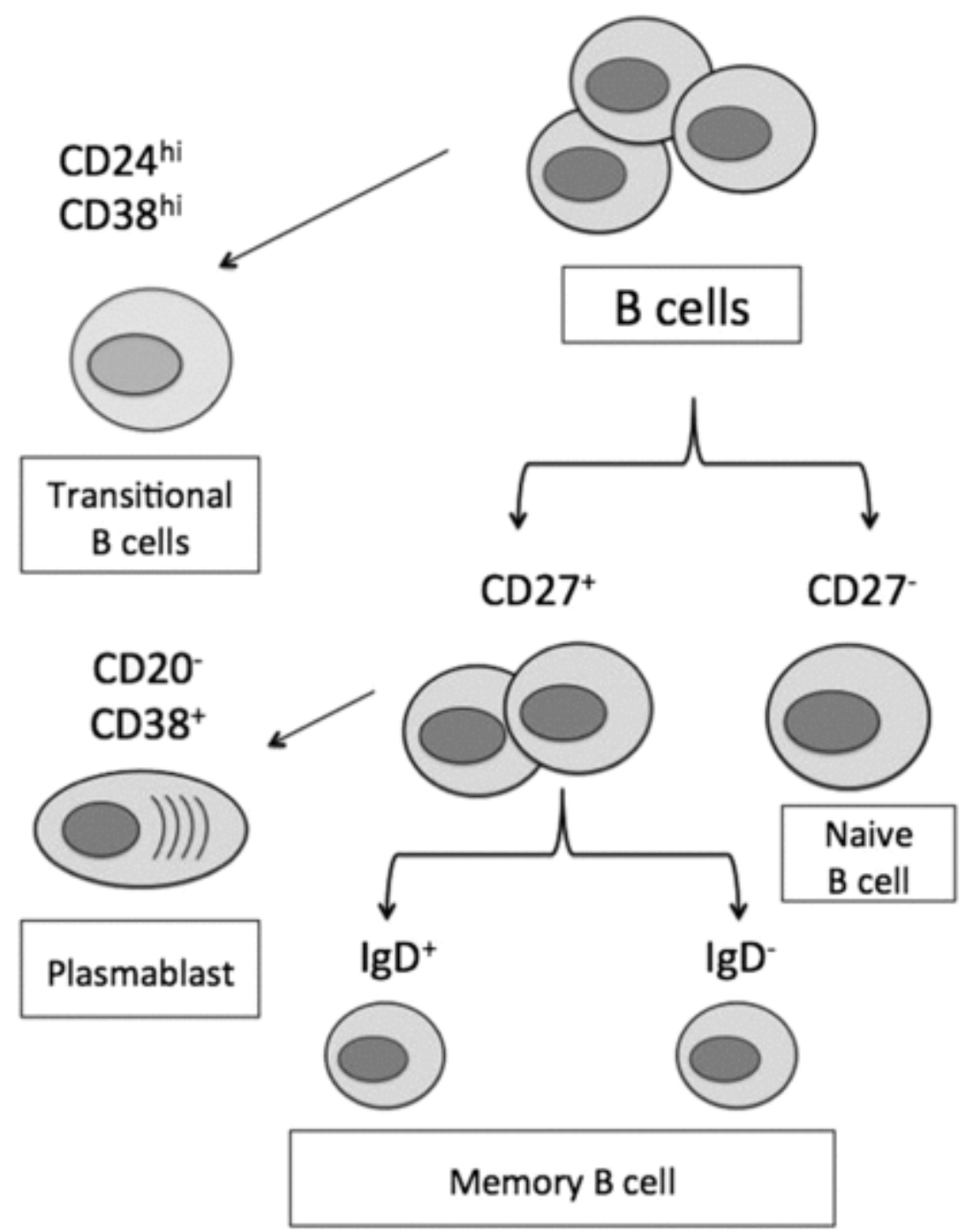

Fig. 2 Identification of B cell subsets suggested by Standardizing immunophenotyping for the Human Immunology Project (Adapted from Maecker et al [40]).

In the setting of transplantation, the ONE Study consortium has recently initiated several clinical trials to evaluate cell therapies oriented to promote tolerance to renal allografts. To compare the value of different cell therapies, the consortium developed a robust immune monitoring strategy. This scheme consisted of establishing standard procedures for whole blood leukocyte subset profiling by flow cytometry: sample collection, storage, preparation, and flow analysis, including protocols for gating subsets. The immunophenotyping consists of six leukocyte profiling panels employing seven to nine surface markers for monitoring T cell, B cell and dendritic cell (DC) subsets, among others. Their B cell panel comprises the use of antibodies for CD45 + leucocytes, CD19 + B cells, CD21 low and CD38 B cells, IgD, IgM, CD24 and CD27. This standardized strategy of leukocyte profiling to identify changes in leukocyte subsets resulted in a high concordance across multiple international sites [41] (Fig. 3). 


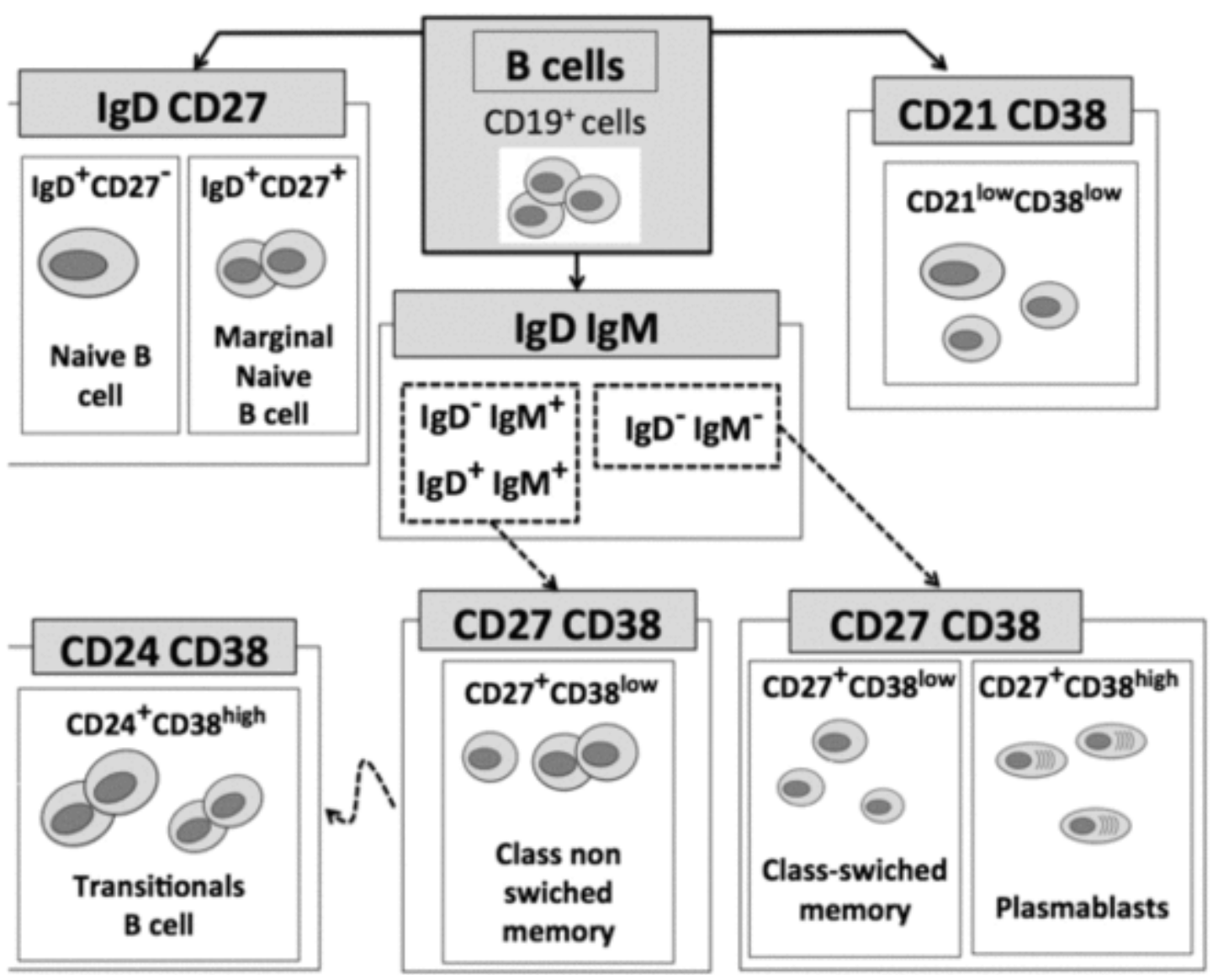

Fig. 3 B cell subset in the One Study: Overview of the gating strategy (Adapted from Streitz et al. [41]).

In summary, characterization of B cells is getting more complex as the knowledge of phenotypes, functionality and clinical engagement increases. It is therefore pivotal to obtain standardized panels that may be reproducible in different laboratories. For the transplant setting, efforts are now being made to harmonize panels and protocols, which should result in a uniform data collection and analysis approach.

\subsection{B cell subtypes and allograft rejection}

The role of B cells in allograft rejection, besides antibody production, is becoming increasingly obvious [42], but their specific role in rejection is still not clear. In the rejection process, B cells may participate through antigen presentation, cytokine production, immune regulation and differentiation into alloantibody-producing plasma cells [43]. The fact that B cells can be involved in antigen presentation during acute cellular rejection has been shown in animal models [44]. Several clinical studies have shown $\mathrm{CD} 20^{+} \mathrm{B}$ cell infiltrates in kidney biopsy samples from patients with acute rejection [45-49]. The absence of $\mathrm{C} 4 \mathrm{~d}$ deposits in peritubular capillaries and circulating antibodies suggested that B cells may have had an antibody-independent function, such as antigen presentation[45]. Additionally, intragraft B cells clusters have been detected in animal models of chronic rejection and explanted transplants lost for chronic rejection [50]. These clusters were the site of intense proliferation and production of antibodies directed against the donor [51,52].

Unlike data on B cell infiltrates in rejecting kidneys, there is not much information about the peripheral B cell subset distribution in kidney transplant recipients [53]. Kamburova et al. has described the kinetics of peripheral $\mathrm{B}$ cells according to the Bm1-Bm5 classification in a cohort of kidney transplant recipients on standard triple immunosuppressive therapy, as well as a group of patients receiving rituximab induction therapy [54]. Patient on standard triple immunosuppressive therapy showed 
a modest decrease in virgin-naïve (Bm2) B-cells post-transplant accompanied with an increase in memory-like B cells [55]. This was accompanied by a rapid and long-lasting decline in Bm2' or transitional B cells after transplantation, which has subsequently been confirmed by an independent study [42]. Even less is known about the peripheral B cell subset distribution upon rejection. Van de Berg et al. found no differences in B cell subset distribution between stable graft recipients and patients with either subclinical or acute rejection by staining for $\operatorname{IgD}$ and $\mathrm{CD} 27$ [56]. Whether changes in subset distribution would be detectable by using additional B cell markers remain unknown. Recently, Nouël and col. have found that kidney transplant patients with chronic antibody-mediated rejection compared with healthy volunteers shows a reduction in the "activated B cells/memory B cells" ratio in peripheral blood, derived from an expansion of Bm5 and a decrease in Bm2 + Bm2' compartment [57]. Patients with rejection showed an increase in memory post-switched B cells, which may be due to continuous stimulation by alloantigens, or an altered B cell differentiation in the germinal centers $[51,52]$.

\subsection{Regulatory $B$ cells and solid organ transplantation}

Paradoxically, B cells and plasma cells have recently been described as being able to regulate immune responses [58,59]. Original findings on the capacity of B cells to regulate came from the field of autoimmunity. For example, when B cell deficient mice were used in the EAE model for multiple sclerosis, disease was still induced, but unlike wild type mice, knockout mice did not go into remission [60]. This indicated that $\mathrm{B}$ cells could potentially be involved in immune modulation, secondary to immune activation. Later, it was shown that B cell mediated regulation in this setting was IL-10 dependent [61].

Up to date, regulatory B cells (Breg) remain a difficult to define population of cells, since the only definite marker at the moment is their capacity to produce IL-10. No master transcriptional regulator, or exclusive phenotype has been described yet. However, there are some phenotypes known in which Breg are enriched. In mice, a phenotype-independent marker for Breg appears to be TIM-1, which strongly enriches for Breg [62,63]. Another B cell population with regulatory properties has been called B10, according to its production of IL-10[64]. In mice, these cells are enriched in the CD1 $\mathrm{d}^{\text {hi }} \mathrm{CD} 5^{+} \mathrm{B}$ cell population [65], whereas in humans these cells are enriched in a memory-like pool of $\mathrm{CD} 27^{+} \mathrm{CD} 24^{\text {hi }} \mathrm{B}$ cells [66]. A second population of human Breg is enriched in the transitional B cell pool of $\mathrm{CD} 24^{\mathrm{hi}} \mathrm{CD} 38^{\text {hi }} \mathrm{B}$ cells $[67,68]$. Interestingly, it has recently been shown that the immunoregulatory cytokines IL-10 and IL-35 could also be produced by CD138 $8^{+}$plasma cells [69], extending the range of B cells capable of regulating immune responses even more.

In transplantation, the notion that B cells may have regulatory properties is a rather new concept. There are a limited number of experimental animal studies that underpin the possibility that Breg may be important for tolerance induction. For TIM-1+ B cells, it has been shown that stimulation with a low affinity anti-TIM-1 antibody resulted in prolonged graft survival in a mouse model of islet transplantation [62]. In concert with these findings, Lee et al. showed that combined anti-CD45RB and anti-TIM-1 treatment resulted in 100\% long-term islet allograft survival [70]. The prolonged graft survival was dependent on the presence of IL-10 producing B cells. The same group recently showed that Breg require the presence of regulatory $\mathrm{T}$ cells (Treg) to establish tolerance, and that the interaction between Breg and Treg is possibly dependent on TGF $\beta$ [71].

Using a mouse model of cardiac transplant tolerance, Le Texier et al. showed that B cells in tolerant animals were blocked in their progression from $\operatorname{IgM}$ to $\operatorname{IgG}$, as well as being negative for the 
memory marker CD27 [72], which is in line with evidence showing that immature B cells have immunoregulatory properties [67].

Clinical evidence for a role of B cells in renal tolerance induction and/or maintenance has also been published [73]. A major breakthrough in this field was when two independent consortium studies on biomarkers of operational tolerance in renal transplant recipients both showed a clear B cell dominated fingerprint of tolerance [27,28]. In separate studies, operationally tolerance was associated with increased levels of peripheral B cells, with increased numbers of naïve and transitional B cells [27,28,74,75], as well as memory B cells [76], compared to stable renal transplant recipients under immunosuppressive drugs. While operational tolerance showed a different B cell subset distribution compared to stable patients, it was similar to healthy non-transplanted individuals.

There are little functional data on B cell regulation in humans. However, when transitional B cells were polyclonally activated, there was a tendency to higher frequencies of IL-10 producing cells in operationally tolerant individuals[27]. In concordance with these findings, increased IL-10 production in total B cells of tolerant individuals has also been observed by the Nantes group [75].

In addition to phenotypic data, gene expression analysis revealed that MS4A1 (CD20) levels in urine were elevated in tolerant patients, whereas the peripheral blood gene expression profile was characterized by high expression of several B cell-related genes. Two studies have since shown that the most differentially expressed genes, elevated during tolerance (MS4A1, CD79B and TCL1A), were expressed at low levels in patients with acute rejection [42,77]. These findings indicate that MS4A1, CD79B and TCL1A may be markers for immune activation/regulation.

The relative role of $\mathrm{B}$ cells to tolerance induction and or maintenance is subject of intense research. With a better understanding on the regulatory capacities and pathways of B cells, targeted therapy to induce regulation may be possible in the future.

\section{Evaluating B cell functionality}

Currently, the few above-mentioned studies on B cell immunophenotyping have not proven to identify patients with a clear risk profile for rejection. The transplant community searches for assays that may predict clinical events in solid-organ transplant recipients with high specificity. As such, detecting antigen-specific immune cells may allow to do so. Therefore, there has been increasing interest in developing strategies to track donor antigen-reactive B cells. This approach may be useful to improve understanding of anti-donor memory responses and should help to get better diagnostic and treatment options in transplant recipients [78]. Studies on HLA-specific B cells are evolving from evaluating the ability of B cells to produce HLA antibodies in vitro to quantifying HLA-specific B cells by flow cytometry and ELISPOT.

\subsection{In vitro assays to evaluate B cell production of HLA specific antibodies}

Activation of B cells is a multistep process, which starts with B cell recognition of its cognate antigen, and leads to the formation of short-lived plasmablasts and, in case of germinal center formation, long-lived plasma cells and memory B cells. In the 90's some research groups were able to grow B cells in culture and differentiate them into plasma cells [79-81]. Other groups had demonstrated the ability to detect and measure precursor frequencies of antibody secreting B lymphocytes specific for several antigens, such as A or B antigens in individuals with different blood groups, rheumatoid factor in patients 
with rheumatoid arthritis or antigens from Plasmodium falciparum in patients with malaria in limiting dilution format cultures [82,83].

Analysis of IgG antibody specificities in supernatants of B cells that are activated in a polyclonal fashion, not leading to in vitro class switching, provides information on the composition of the preexisting memory B cell compartment. In the setting of transplantation, Mulder and colleagues were the first to describe an assay activating $\mathrm{B}$ cells in vitro and analyzing their HLA antibody secreting capacity [84]. They were able to calculate the precursor frequency of B-cells with HLA specificity by measuring HLA antibodies in the supernatants of limiting dilution B cell cultures. These studies permitted them to dissect the specificities of circulating B cells in patients, as well as to study the correlation with serum antibodies.

More recently, Han and colleagues used purified B cells from allograft recipients, sensitized patients or multiparous women and cultured them with polyclonal stimulation to obtain supernatants for studying HLA antibody specificities with Luminex. Interestingly, they found that donor-specific antibodies were sometimes produced in B-cell cultures when circulating HLA antibodies were negative [85]. Using a similar approach, Snanoudj and colleagues tested the presence of circulating alloreactive memory B cells in 69 patients with end-stage renal disease under renal replacement therapy. They showed HLA-specific memory B cells in patients with serum HLA antibodies following multiple HLA-immunizing events. In other patients, serum HLA antibodies were not associated with the detection of specific memory B cells [86]. In concordance with the study of Han et al., some specificities found in the culture supernatant were not detected in the accompanying serum sample, indicating that memory B cells may be present in the absence of antibody production.

\subsection{Flow cytometry based assays for HLA specific B-cell quantification}

Besides phenotypic characterization, flow cytometry has also been used with the intention to quantify the peripheral HLA-specific B-cell compartment. The Leiden group was able to quantify HLAspecific B cells by flow cytometry, staining CD19+ B-cells with HLA tetramers (HLA antigens) without pre-culturing those B-cells [87]. Using developed human B hybridomas specific for HLA molecules, they proved that tetrameric HLA-A2 provided a suitable affinity ligand for isolation of allospecific B cells. They then isolated HLA-specific B cells by HLA tetramers from HLA-immunized individuals and induced those cells to produce HLA-A2 antibodies in culture. The group of Zachary at Johns Hopkins confirmed the capacity to measure peripheral HLA specific B cells in kidney transplant recipients by staining with HLA tetramers. They used lymphocyte preparations enriched for B cells and stained them with three different HLA tetramers and found that the number and phenotype of HLA-specific B cells (tetramer stained B cells) correlated with current or historic HLA sensitization [88,89]. CD27 ${ }^{+}$B cells were more frequent among tetramer positive B cells than among general B cells, but the proportion of CD $38^{+}$B cells was similar. Subsequently, the same group showed that patients without circulating HLA antibodies in pre-transplant sera, but considered sensitized based on the frequencies of peripheral HLAspecific B cells, developed post-transplant antibodies against the tetramer antigen if untreated with rituximab [90].

Tetramer technology for the detection of HLA-specific B cells is a convenient method, but suffers from the presence of B cells that can recognize streptavidin-biotin or the fluorochrome, both of which are part of the HLA tetramer complex. [91,92] Pre-incubation of B cells with streptavidin-biotin [87]and/or the use of multiple tetramers coupled to different fluorochromes [93] can be used to overcome these problems. 


\subsection{Toward ELISPOT}

The ELISPOT assay was originally developed as a modification of ELISA techniques to detect antigen-specific antibody-secreting B cells [94]. It is a solid-phase enzyme-linked immunosorbent assay designed for enumeration of cells secreting specific antibodies. It employs B cells from immunized animals or humans, which are incubated in antigen-coated polystyrene plates (the antigen of interest). After removal of the cells, bound secreted antibodies are visualized by means of an immunoenzyme procedure. Spots form where antibody production has occurred and can be enumerated to establish a frequency of antigen-specific B cells. ELISPOT has been adapted for various purposes, especially to identify and enumerate cells able to produce cytokines. The high sensitivity of the assay could be useful for detecting low-frequency HLA-specific B-cells.

The group of Mayo Clinic published the first report of a B cell ELISPOT applied to evaluate HLA antibody producing cells in 2008 [95]. They selected the CD138 $8^{+}$plasma cell fraction of the bone marrow for the assay, which mainly expressed the long-lived PC phenotype (CD138 $8^{+}$CD $38^{+}$, immunoglobulin $\left.{ }^{+}\right)$. The correlation between serum alloantibody and plasma cell alloantibody specificity was generally high. However, the correlation between allospecific plasma cell frequency in the bone marrow and serum alloantibody levels measured by the single antigen flow bead assay was poor. The investigators did not find that pretransplant treatment with plasma-exchange, intravenous immunoglobulin or polyclonal rabbit antithymocyte globulin had an effect on the number of HLA antibody producing plasma cells or alloantibody production. The need of an invasive procedure to obtain bone marrow samples has limited this approach.

In contrast, the Leiden group developed an ELISPOT assay using peripheral blood B-cells, which is a more realistic source for an assay to monitor patients. Working with B-cells poses an additional challenge, since they require in vitro pre-activation, as described above. Nevertheless, the group developed an assay that allowed for determining the frequency of HLA class I-specific memory B-cells from peripheral blood [96]. Monomeric HLA class I molecules were used as antigen in the ELISPOT plates (Fig. 4, left panel). Exceptional validation of the technique was possible for the availability of Bcell hybridomas, which produce antibodies toward defined HLA molecules [97]. They reported on the detection of HLA-specific B-cells in pregnancy-immunized individuals and in immunized kidney transplant recipients. Recently, the same group has developed a similar assay for the detection of HLA class II specific memory B cells [98]. Analogous to the HLA class I assay, this technique uses monomeric HLA class II molecules for the detection of HLA-specific memory B cells (Fig. 4, right panel). This HLA class II specific memory B cell ELISPOT assay may be of great clinical interest, since HLA class II molecules are predominantly implicated in chronic antibody-mediated rejection [10,11,29,99]. 
HLA class I ELISPOT
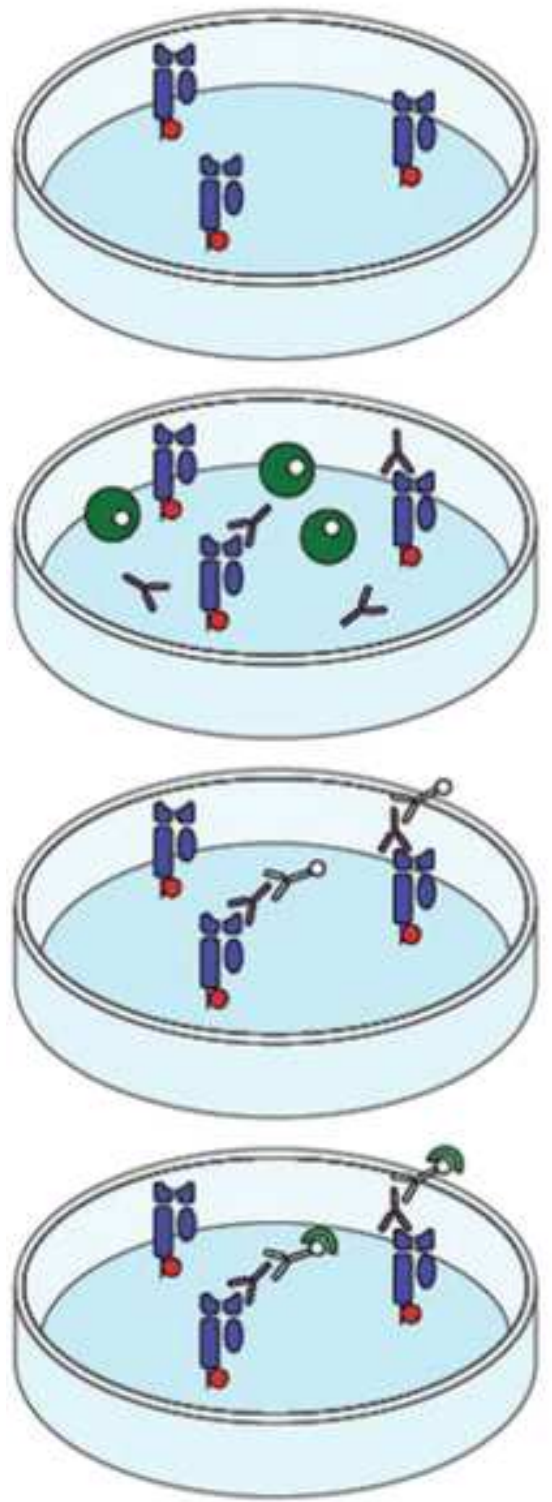

1. Coating

4. Washing
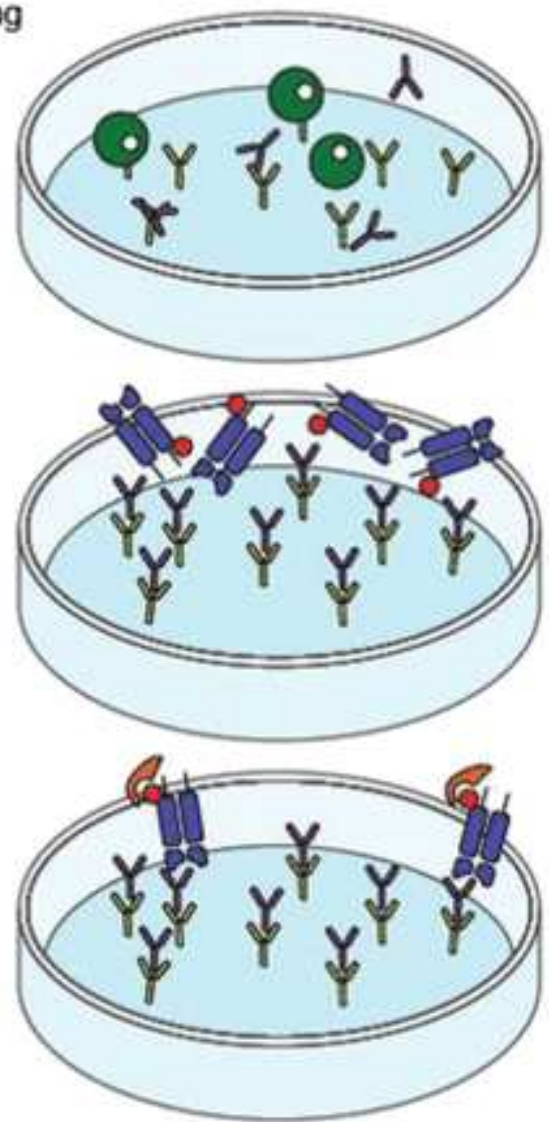

Legend

\section{0}

Biotinylated HLA class I molecules

- Activated, $A b$ producing $B$ cells

$\checkmark$ Antibody produced by activated $B$ cell

\section{$\gamma^{\circ}$ HRP labeled anti-human IgG mAb}

3. Cell incubation

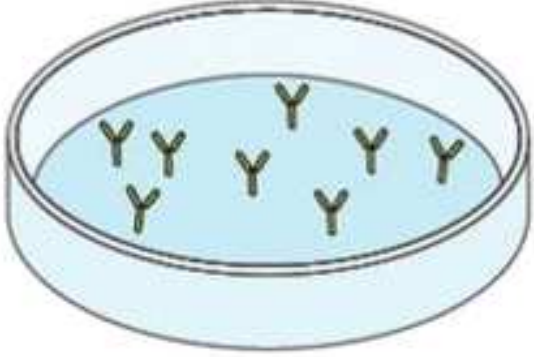

2. Washing/blocking

5. Detection

6. Washing

7. Visualization

HLA class \| ELISPOT

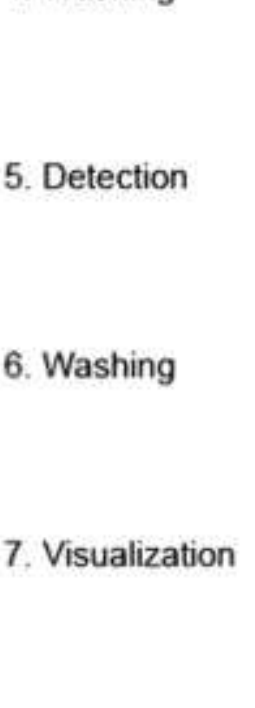

S substrate

$Y$ anti-human $\lg G$ coating $\mathrm{mAb}$

it

Biotinylated HLA class II molecules

\section{Streptavidin ALP / substrate}

Fig. 4 Schematic overview of HLA class I- and HLA class II-specific memory B cell ELISPOT assays (left panel) ELISPOT plates are first coated with streptavidin followed by incubation of saturating levels of recombinant biotinylated HLA class I molecules. Following a blocking step, pre-activated, antibody producing B cells are added and left overnight. Then unbound antibodies as well as the B cells are washed away, leaving bound antibodies as footprints of HLA-class I antibody-producing cells. Bound antibodies are detected by a horseradish peroxidase (HRP)-labeled anti-human IgG monoclonal antibody. Finally, using a substrate, footprints of the HLA class I antibody producing cell can be visualized as individual 
spots. (Right panel) ELISPOT plates are first coated with an anti-human IgG monoclonal antibody at saturating levels. Following blocking of the ELISPOT plate, pre-activated, antibody producing B cells are added and left overnight. Cells are then washed away leaving all IgG antibodies produced by the activated B cells bound to the plate. Among these bound antibodies, the HLA-specific antibodies can be detected by adding biotinylated HLA class II molecules. Finally, using streptavidin-alkaline phosphatase (ALP) and a proper substrate, the footprint of each HLA class II antibody-producing cell can be visualized as a single spot.

Despite the elegance of being able to detect HLA-specific memory B cells towards single defined specificities, the ideal clinical test should offer the possibility to detect the total array of donor-specific memory B cells. Lynch and colleagues from University of Michigan have recently described a donorspecific B cell ELISPOT for HLA class I [26]. They cultured isolated donor fibroblasts as monolayers for the detection of donor-specific HLA-class I-specific memory B-cells in ELISPOT format. Testing nine subjects without detectable donor-specific antibodies before or after transplantation, they found that all patients had detectable HLA-specific B cells eight weeks after transplantation. The responses were directed against the donor HLA-class I antigens. From a clinical point of view, this assay faces serious difficulties as preparing viable donor fibroblasts for the assay needs extensive culture periods.

The above-mentioned functional B cell assays as yet remain in the research phase and need clinical validation and well as reproduction by independent laboratories. Although assaying the peripheral blood for HLA-specific memory B cells is useful and practically feasible, one has to keep in mind that peripheral HLA specific B cells are low in numbers and may not reflect the complete alloimmune response, which may be predominantly active locally in the graft or secondary lymphoid organs [19,51,52].

\section{Summary}

The knowledge and new approaches into phenotypic and functional heterogeneity of B cells have several implications for transplantation. It is currently accepted that the role of $\mathrm{B}$ cells in alloimmune response is not limited to alloantibody production. Distinct B cell subsets and their descendants may differ in their cytokine production and surface molecule expression, factors that in turn may influence their antigen-presenting or immune regulatory functions. The kinetics of donor-specific B cells in transplant recipients remains currently unknown. The difference in the activation of B cells or the affinity of antibodies generated by different memory B cells and plasma cells may determine the pathogenicity of resulting donor-reactive antibodies. Besides, it is unclear if different B cell subpopulations may vary in their susceptibility to maintenance immunosuppressive or B cell specific targeted therapies.

\section{Acknowledgements}

This study was performed with funding from the projects PI10/01370, PI13/00598 SpanishMinistry of Health ISCIII FIS-FEDER, Marato TV3 137/C/2012 and RedinRen RD12/0021/0024.

\section{References}

[1] R. Patel and P.I. Terasaki, Significance of the positive crossmatch test in kidney transplantation, $N$ Engl J Med 280, 1969, 735-739.

[2] H.U. Meier-Kriesche, J.D. Schold and B. Kaplan, Long-term renal allograft survival: have we made significant progress or is it time to rethink our analytic and therapeutic strategies?, Am J Transplant 4, 2004, 1289-1295. 
[3] B.L. Kasiske, R.S. Gaston, S. Gourishankar, et al., Long-term deterioration of kidney allograft function, Am J Transplant 5, 2005, 1405-1414.

[4] F. Moreso and D. Hernandez, Has the survival of the graft improved after renal transplantation in the era of modern immunosuppression?,Nefrologia 33, 2013, 14-26.

[5] D.L. Roelen, I.I. Doxiadis and F.H. Claas, Detection and clinical relevance of donor specific HLA antibodies: a matter of debate, Transpl Int 25, 2012,604-610.

[6] S. Mohan, A. Palanisamy, D. Tsapepas, et al., Donor-specific antibodies adversely affect kidney allograft outcomes, J Am Soc Nephrol 23, 2012,2061-2071.

[7] M. Crespo, A. Torio, V. Mas, et al., Clinical relevance of pretransplant anti-HLA donor-specific antibodies: does C1q-fixation matter?, Transpl Immunol 29, 2013, 28-33.

[8] K. Marfo, M. Ajaimy, A. Colovai, et al., Pretransplant immunologic risk assessment of kidney transplant recipients with donor-specific anti-human leukocyte antigen antibodies, Transplantation 98, 2014, 1082 1088 .

[9] G. Hönger, H. Hopfer, M.L. Arnold, B.M. Spriewald, S. Schaub and P. Amico,Pretransplant IgG subclasses of donor-specific human leukocyte antigen antibodies and development of antibody-mediated rejection,Transplantation 92, 2011, 41-47.

[10] M. Crespo, M. Pascual, N. Tolkoff-Rubin, et al., Acute humoral rejection in renal allograft recipients: I. Incidence, serology and clinical characteristics,Transplantation 71, 2001, 652-658.

[11] C. Wiebe, I.W. Gibson, T.D. Blydt-Hansen, et al., Evolution and clinical pathologic correlations of de novo donor-specific HLA antibody post kidney transplant, Am J Transplant 12, 2012, 1157-1167.

[12] M.J. Everly, L.M. Rebellato, C.E. Haisch, et al., Incidence and impact of de novo donor-specific alloantibody in primary renal allografts,Transplantation 95, 2013, 410-417.

[13] J. Pascual, M.J. Pérez-Sáez, M. Mir and M. Crespo, Chronic renal allograft injury: early detection, accurate diagnosis and management, Transplant Rev (Orlando) 26, 2012, 280-290.

[14] L.C. Racusen, R.B. Colvin, K. Solez, et al., Antibody-mediated rejection criteria—an addition to the Banff 97 classification of renal allograft rejection, Am J Transplant 3, 2003, 708-714.

[15] J.D. Smith, N.R. Banner, I.M. Hamour, et al., De novo donor HLA-specific antibodies after heart transplantation are an independent predictor of poor patient survival, Am J Transplant 11, 2011, 312-319.

[16] L.D. Snyder, Z. Wang, D.-F. Chen, et al., Implications for human leukocyte antigen antibodies after lung transplantation: a 10-year experience in 441 patients, Chest 144, 2013, 226-233.

[17] J. Pascual, M.D. Samaniego, J.R. Torrealba, et al., Antibody-mediated rejection of the kidney after simultaneous pancreas-kidney transplantation,J Am Soc Nephrol 19, 2008, 812-824.

[18] H. Kaneku, J.G. O'Leary, N. Banuelos, et al., De novo donor-specific HLA antibodies decrease patient and graft survival in liver transplant recipients,Am J Transplant 13, 2013, 1541-1548.

[19] T. Bachelet, L. Couzi, S. Lepreux, et al., Kidney intragraft donor-specific antibodies as determinant of antibody-mediated lesions and poor graft outcome, Am J Transplant 13, 2013, 2855-2864.

[20] B. Sis, G.S. Jhangri, J. Riopel, et al., A new diagnostic algorithm for antibody-mediated microcirculation inflammation in kidney transplants,Am J Transplant 12, 2012, 1168-1179. 
[21] M. Haas, B. Sis, L.C. Racusen, et al., Banff 2013 meeting report: inclusion of c4d-negative antibodymediated rejection and antibody-associated arterial lesions, Am J Transplant 14, 2014, 272-283.

[22] J. Sellarés, J. Reeve, A. Loupy, et al., Molecular diagnosis of antibody mediated rejection in human kidney transplants, Am J Transplant 13, 2013,971-983.

[23] Olivier Thaunat, Humoral immunity in chronic allograft rejection: puzzle pieces come together, Transpl Immunol 26, 2012, 101-106.

[24] J. Sellares, D.G. de Freitas, M. Mengel, et al., Understanding the causes of kidney transplant failure: the dominant role of antibody-mediated rejection and nonadherence, Am J Transplant 12, 2012, 388-399.

[25] A. Loupy, C. Lefaucheur, D. Vernerey, C. Prugger, J.P. Duong van Huyenand N. Mooney, Complementbinding anti-HLA antibodies and kidney-allograft survival, N Engl J Med 369, 2013, 1215-1226.

[26] R.J. Lynch, I.A. Silva, B.J. Chen, J.D. Punch, M. Cascalho and J.L. Platt,Cryptic B cell response to renal transplantation, Am J Transplant 13, 2013,1713-1723.

[27] K.A. Newell, A. Asare, A.O. Kirk, et al., Identification of a B cell signature associated with renal transplant tolerance in humans, J Clin Invest 120, 2010, 1836.

[28] P. Sagoo, E. Perucha, B. Sawitzki, et al., Development of a cross-platform biomarker signature to detect renal transplant tolerance in humans, J Clin Invest 120, 2010, 1848.

[29] M. Crespo, J. Yelamos, D. Redondo, et al., Circulating NK-cell subsets in renal allograft recipients with anti-HLA donor-specific antibodies, Am J Transplant 2015, [E-Pub].

[30] D.K. Kwan and A. Norman, Letter: identification of two populations of human lymphocytes, Acta Cytol 18, 1974, 189-191.

[31] T.W. LeBien and T.F. Tedder, B lymphocytes: how they develop and function, Blood 112, 2008, 15701580 .

[32] I. Sanz, C. Wei, F.E. Lee and J. Anolik, Phenotypic and functional heterogeneity of human memory B cells, Semin Immunol 20, 2008, 67-82.

[33] V. Pascual, Y.J. Liu, A. Magalski, O. de Bouteiller, J. Banchereau and J.D.Capra, Analysis of somatic mutation in five B cell subsets of human tonsil, J Exp Med 180, 1994, 329-339.

[34] S.M. Jackson, P.C. Wilson, J.A. James and J.D. Capra, Human B cell subsets, Adv Immunol 98, 2008, 151224.

[35] J. Bohnhorst, M.B. Bjorgan, J.E. Thoen, J.B. Natvig and K.M. Thompson,Bm1-Bm5 classification of peripheral blood B cells reveals circulating germinal center founder cells in healthy individuals and disturbance in the B cell subpopulations in patients with primary Sjögren's syndrome, $J$

Immunol 167, 2001, 3610-3618.

[36] U. Klein, K. Rajewsky and R. Küppers, Human immunoglobulin (Ig)M + IgD + peripheral blood B cells expressing the CD27 cell surface antigen carry somatically mutated variable region genes: CD27 as a general marker for somatically mutated (memory) B cells, J Exp Med 188, 1998, 1679-1689.

[37] K. Agematsu, S. Hokibara, H. Nagumo and A. Komiyama, CD27: a memory B-cell marker, Immunol Today 21, 2000, 204-206. 
[38] J.F. Fecteau, G. Côté and S. Néron, A new memory CD27-IgG + B cell population in peripheral blood expressing VH genes with low frequency of somatic mutation, J Immunol 177, 2006, 3728-3736.

[39] D.A. Kaminski, C. Wei, Y. Qian, A.F. Rosenberg and I. Sanz, Advances in human B cell phenotypic profiling, Front Immunol 3, 2012 Oct 10, 302,http://dx.doi.org/10.3389/fimmu.2012.00302. eCollection 2012.

[40] H.T. Maecker, J.P. McCoy and R. Nussenblatt, Standardizing immunophenotyping for the Human Immunology Project, Nat Rev Immunol 12, 2012, 191-200.

[41] M. Streitz, T. Miloud, M. Kapinsky, et al., Standardization of whole blood immune phenotype monitoring for clinical trials: panels and methods from the ONE study, Transplant Res 2, 2013, 17.

[42] S. Heidt, M. Vergunst, J.D. Anholts, et al., B cell markers of operational tolerance can discriminate acute kidney allograft rejection from stable graft function, Transplantation 2014, [Epub ahead of print].

[43] H. Noorchashm, A.J. Reed, S.Y. Rostami, et al., B cell-mediated antigen presentation is required for the pathogenesis of acute cardiac allograft rejection, J Immunol 177, 2006 Dec 1, 7715-7722.

[44] Y.H. Ng, M.H. Oberbarnscheidt, H.C. Chandramoorthy, R. Hoffman and G.Chalasani, B cells help alloreactive T cells differentiate into memory T cells,Am J Transplant 10, 2010, 1970-1980.

[45] M. Sarwal, M.S. Chua, N. Kambham, et al., Molecular heterogeneity in acute renal allograft rejection identified by DNA microarray profiling, $N$ Engl J Med 349, 2003, 125-138.

[46] E.W. Tsai, P. Rianthavorn, D.W. Gjertson, W.D. Wallace, E.F. Reed and R.B.Ettenger, CD20 + lymphocytes in renal allografts are associated with poor graft survival in pediatric patients, Transplantation 82, 2006, 1769-1773.

[47] O.M. Steinmetz, U. Panzer, U. Kneissler, et al., BCA-1/CXCL13 expression is associated with CXCR5positive B-cell cluster formation in acute renal transplant rejection, Kidney Int 67, 2005, 1616-1621.

[48] C. Doria, F. di Francesco, C.B. Ramirez, et al., The presence of B-cell nodules does not necessarily portend a less favorable outcome to therapy in patients with acute cellular rejection of a renal allograft, Transplant Proc 38, 2006,3441-3444.

[49] S.M. Bagnasco, W. Tsai, M.H. Rahman, et al., CD20-positive infiltrates in renal allograft biopsies with acute cellular rejection are not associated with worse graft survival, Am J Transplant 7, 2007, 1968-1973.

[50] V. Zarkhin, N. Kambham, L. Li, et al., Characterization of intra-graft B cells during renal allograft rejection, Kidney Int 74, 2008, 664-673.

[51] O. Thaunat, A.C. Field, J. Dai, et al., Lymphoid neogenesis in chronic rejection: evidence for a local humoral alloimmune response, Proc Natl Acad Sci U S A 102, 2005, 14723-14728.

[52] O. Thaunat, N. Patey, G. Caligiuri, et al., Chronic rejection triggers the development of an aggressive intragraft immune response through recapitulation of lymphoid organogenesis, $J$ Immunol 185, 2010, 717-728.

[53] V. Zarkhin, G. Chalasani and M.M. Sarwal, The yin and yang of B cells in graft rejection and tolerance, Transplant Rev 24, 2010, 67-78.

[54] E.G. Kamburova, H.J. Koenen, M.W. van den Hoogen, M.C. Baas, I. Joostenand L.B. Hilbrands, Longitudinal analysis of T and B cell phenotype and 
function in renal transplant recipients with or without rituximab induction therapy, PLoS One 9, 2014 Nov 13, e112658.

[55] V. Zarkhin and M.M. Sarwal, The coin toss of B cells in rejection and tolerance: danger versus defense, Semin Immunol 24, 2012, 86-91.

[56] P.J. van de Berg, E.C. Hoevenaars, S.L. Yong, et al., Circulating lymphocyte subsets in different clinical situations after renal transplantation,Immunology 136, 2012, 198-207.

[57] A. Nouël, I. Ségalen, C. Jamin, et al., B cells display an abnormal distribution and an impaired suppressive function in patients with chronic antibody-mediated rejection, Kidney Int 85, 2014, 590-599.

[58] J. Stolp, L.A. Turka and K.J. Wood, B cells with immune-regulating function in transplantation, Nat Rev Nephrol 10, 2014, 389-397.

[59] E.S. Woodle and D.M. Rothstein, Clinical implications of basic science discoveries: Janus resurrectedtwo faces of B cell and plasma cell biology,Am J Transplant 15, 2015, 39-43.

[60] S.D. Wolf, B.N. Dittel, F. Hardardottir and C.A. Janeway, Jr., Experimental autoimmune encephalomyelitis induction in genetically B cell-deficient mice, J Exp Med 184, 1996, 2271-2278.

[61] S. Fillatreau, C.H. Sweenie, M.J. McGeachy, D. Gray and S.M. Anderton, B cells regulate autoimmunity by provision of IL-10, Nat Immunol 3, 2002,944-950.

[62] Q. Ding, M. Yeung, G. Camirand, et al., Regulatory B cells are identified by expression of TIM-1 and can be induced through TIM-1 ligation to promote tolerance in mice, J Clin Invest 121, 2011, 3645-3656.

[63] M.Y. Yeung, Q. Ding, C.R. Brooks, et al., TIM-1 signaling is required for maintenance and induction of regulatory B cells, Am J Transplant 2015,http://dx.doi.org/10.1111/ajt.13087, [Epub ahead of print].

[64] I. Kalampokis, A. Yoshizaki and T.F. Tedde, IL-10-producing regulatory B cells (B10 cells) in autoimmune disease, Arthritis Res Ther 1, 2013, S1.

[65] K. Yanaba, J.D. Bouaziz, K.M. Haas, J.C. Poe, M. Fujimoto and T.F. Tedder,A regulatory B cell subset with a unique CD1dhiCD5 + phenotype controls T cell-dependent inflammatory responses, Immunity 28, 2008, 639-650.

[66] Y. Iwata, T. Matsushita, M. Horikawa, et al., Characterization of a rare IL-10-competent B-cell subset in humans that parallels mouse regulatory B10 cells, Blood 117, 2011, 530-541.

[67] P.A. Blair, L.Y. Norena, F. Flores-Borja, et al., CD19(+)CD24(hi)CD38(hi) B cells exhibit regulatory capacity in healthy individuals but are functionally impaired in systemic lupus erythematosus patients, Immunity 32, 2010,129-140.

[68] F. Flores-Borja, A. Bosma, D. Ng, et al., CD19 + CD24hiCD38hi B cells maintain regulatory T cells while limiting TH1 and TH17 differentiation, Sci Transl Med 5, 2013, 173ra123.

[69] P. Shen, T. Roch, V. Lampropoulou, et al., IL-35-producing B cells are critical regulators of immunity during autoimmune and infectious diseases,Nature 507, 2014, 366-370.

[70] K.M. Lee, J.I. Kim, R. Stott, et al., Anti-CD45RB/anti-TIM-1-induced tolerance requires regulatory B cells, Am J Transplant 12, 2012,2072-2078.

[71] K.M. Lee, R.T. Stott, G. Zhao, et al., TGF-beta-producing regulatory B cells induce regulatory T cells and promote transplantation tolerance, Eur J Immunol 2014. 
[72] L. Le Texier, P. Thebault, A. Lavault, et al., Long-term allograft tolerance is characterized by the accumulation of B cells exhibiting an inhibited profile,Am J Transplant 11, 2011, 429-438.

[73] S. Heidt and K.J. Wood, Biomarkers of operational tolerance in solid organ transplantation, Exp Opin Med Diagn 6, 2012, 281-293.

[74] H.M. Silva, M.C. Takenaka, P.M. Moraes-Vieira, et al., Preserving the B cell compartment favors operational tolerance in human renal transplantation,Mol Med 18, 2012, 733-743.

[75] M. Chesneau, A. Pallier, F. Braza, et al., Unique B cell differentiation profile in tolerant kidney transplant patients, Am J Transplant 14, 2014, 144-155.

[76] A. Pallier, S. Hillion, R. Danger, et al., Patients with drug-free long-term graft function display increased numbers of peripheral B cells with a memory and inhibitory phenotype, Kidney Int 78, 2010, 503-513.

[77] O. Viklicky, E. Krystufkova, I. Brabcova, et al., B-cell-related biomarkers of tolerance are up-regulated in rejection-free kidney transplant recipients,Transplantation 95, 2013, 148-154.

[78] A. Valujskikh and J.S. Bromberg, Literature watch: implications for transplantation, Am J Transplant 13, 2013, 1117.

[79] J. Banchereau and F. Rousset, Growing human B lymphocytes in the CD40 system, Nature 353, 1991, 678-679.

[80] F. Rousset, S. Peyrol, E. Garcia, et al., Long-term cultured CD40-activated B lymphocytes differentiate into plasma cells in response to IL-10 but not IL-4, Int Immunol 7, 1995, 1243-1253.

[81] R.J. Armitage, B.M. Macduff, M.K. Spriggs and W.C. Fanslow, Human B cell proliferation and Ig secretion induced by recombinant CD40 ligand are modulated by soluble cytokines, $J$ Immunol 150, 1993, 3671-3680.

[82] R. Rieben, A. Tucci, U.E. Nydegger and R.H. Zubler, Self tolerance to human A and B histo-blood group antigens exists at the B cell level and cannot be broken by potent polyclonal B cell activation in vitro, Eur J Immunol 22, 1992, 2713-2717.

[83] T.L. Vischer, C.F. Werner-Favre, L. Wen and R.H. Zubler, Quantitative analysis of precursors frequency of rheumatoid factor (RF) producing human B cells, Scand J Rheumatol Suppl 75, 1988, 123-126.

[84] A. Mulder, M.J. Kardol, J. Kamp, et al., Determination of the frequency of HLA antibody secreting Blymphocytes in alloantigen sensitized individuals, Clin Exp Immunol 124, 2001, 9-15.

[85] M. Han, J. Rogers, B. Lavingia, et al., Peripheral blood B cells producing donor-specific HLA antibodies in vitro, Hum Immunol 70, 2009, 29-34.

[86] R. Snanoudj, F.H. Claas, S. Heidt, C. Legendre, L. Chatenoud and S.Candon, Restricted specificity of peripheral alloreactive memory B cells in HLA-sensitized patients awaiting a kidney transplant, Kidney Int 2015.

[87] A. Mulder, C. Eijsink, M.J. Kardol and P. Stastny, Identification, isolation, and culture of HLA-A2-specific B lymphocytes using MHC class I tetramers,J Immunol 171, 2003, 6599-6603.

[88] A.A. Zachary, D. Kopchaliiska, R.A. Montgomery and M.S. Leffell, HLA-specific B cells: 1. A method for their detection, quantification, and isolation using HLA tetramers, Transplantation 83, 2007, 982-988. 
[89] A.A. Zachary, D. Kopchaliiska, R.A. Montgomery, J.K. Melancon and M.S.Leffell, HLA-specific B cells: 11. Application to transplantation,Transplantation 83, 2007, 989-994.

[90] A.A. Zachary, D.P. Lucas, R.A. Montgomery and M.S. Leffell, Rituximab prevents an anamnestic response in patients with cryptic sensitization to HLA, Transplantation 95, 2013, 701-704.

[91] K.A. Pape, J.J. Taylor, R.W. Maul, P.J. Gearhart and M.K. Jenkins, Different B cell populations mediate early and late memory during an endogenous immune response, Science 331, 2011, 1203-1207.

[92] J.J. Taylor, R.J. Martinez, P.J. Titcombe, et al., Deletion and anergy of polyclonal B cells specific for ubiquitous membrane-bound self-antigen, J Exp Med 209, 2012, 2065-2077.

[93] J. Chen, H. Yin, J. Xu, et al., Reversing endogenous alloreactive B cell GC responses with anti-CD154 or CTLA-4Ig, Am J Transplant 13, 2013,2280-2292.

[94] C.C. Czerkinsky, L.A. Nilsson and H. Nygren, A solid-phase enzyme-linked immunospot (ELISPOT) assay for numeration of specific antibody-secreting cells, J Immunol Methods 65, 1983, 109-121.

[95] D.K. Perry, H.S. Pollinger, J.M. Burns, et al., Two novel assays of alloantibody-secreting cells demonstrating resistance to desensitization with IVIG and rATG, Am J Transplant 8, 2008, 133-143.

[96] S. Heidt, D.L. Roelen, Y.J. de Vaal, et al., A Novel ELlSPOT assay to quantify HLA-specific B cells in HLA-immunized individuals, Am J Transplant 12, 2012, 1469-1478.

[97] A. Mulder, M.J. Kardol, J.S. Arn, et al., Human monoclonal HLA antibodies reveal interspecies cross reactive swine MHC class I epitopes relevant for xenotransplantation, Mol lmmunol 47, 2010, 809-815.

[98] G.E. Karahan, Y.J. de Vaal, D.L. Roelen, R. Buchli, F.H. Claas and S. Heidt,Quantification of HLA class II-specific memory B cells in HLA-sensitized individuals, Hum Immunol 2015, [pii: S01988859(15)00015-4. EPub].

[99] N. El-Awar, A. Nguyen, K. Almeshari, et al., HLA class II DQA and DQB epitopes: recognition of the likely binding sites of HLA-DQ alloantibodies eluted from recombinant HLA-DQ single antigen cell lines, Hum Immunol 74, 2013, 1141-1152 